\title{
Systemic Lupus Erythematosus (SLE) Therapy: The Old and the New
}

Fabio Basta · Federica Fasola - Konstantinos Triantafyllias ·

Andreas Schwarting

Received: April 16, 2020 / Published online: June 2, 2020

(C) The Author(s) 2020

\section{ABSTRACT}

Despite recent improvements in the treatment of systemic lupus erythematosus (SLE), disease activity, comorbidities and drug toxicity significantly contribute to the risk of progressive irreversible damage accrual and increased mortality in patients with this chronic disease. Moreover, even lupus patients in remission often report residual symptoms, such as fatigue, which have a considerable impact on their health-related quality of life. In recent decades, SLE treatment has moved from the use of

Digital Features To view digital features for this article go to: https://doi.org/10.6084/m9.figshare.12288038.

F. Basta $(\bowtie) \cdot$ F. Fasola $\cdot$ K. Triantafyllias .

A. Schwarting

Acura Rheumatology Center Rhineland Palatinate,

Bad Kreuznach, Germany

e-mail: fabiobasta@libero.it

\section{A. Schwarting}

Division of Rheumatology and Clinical

Immunology, University Medical Center, Johannes

Gutenberg University, Mainz, Germany

F. Basta - F. Fasola - K. Triantafyllias - A. Schwarting

University Center of Autoimmunity, Johannes

Gutenberg University, Mainz, Germany hydroxychloroquine, systemic glucocorticosteroids and conventional immunosuppressive drugs to biologic agents, of which belimumab is the first and only biologic agent approved for the treatment for SLE to date. Novel therapies targeting interferons, cytokines and their receptors, intracellular signals, plasma cells, $\mathrm{T}$ lymphocytes and co-stimulatory molecules are being evaluated. In the context of a holistic approach, growing evidence is emerging of the importance of correct lifestyle habits in the management of lupus manifestations and comorbidities. The aim of this paper is to provide an overview of current pharmacological and non-pharmacological treatment options and emerging therapies in SLE.

Keywords: Belimumab; Fatigue; Hydroxychloroquine; Lupus; Lupus nephritis; Management; Rituximab; SLE; Systemic lupus erythematosus; Therapy 


\section{Key Summary Points}

Antimalarials are still considered to be the cornerstone of treatment for systemic lupus erythematosus (SLE).

Glucocorticosteroids are an important component of SLE treatment as they rapidly ablate the autoimmune response in organ-threatening manifestations, but the risk for damage accrual increases considerably at doese of $>5-7.5 \mathrm{mg} /$ day.

Belimumab, a fully humanized monoclonal antibody which inhibits B-lymphocyte stimulator, is the only targeted biologic agent licensed for the treatment of SLE to date.

Adjuvant treatments and nonpharmacological interventions are fundamental in the context of a holistic approach to the treatment of patients with SLE.

Among emerging therapies, anifrolumab, a fully human monoclonal antibody, has shown positive results in a phase III trial, indicating the potential of antiinterferons in the treatment of SLE.

\section{INTRODUCTION}

Systemic lupus erythematosus (SLE; lupus) is a complex autoimmune disease with a chronic relapsing-remitting course and variable manifestations leading to a spectrum of disease ranging from mild to life-threatening illness. The clinical onset of SLE derives from the interaction between genetic predisposition and environmental, immunological and hormonal factors, with a strong predilection for women of childbearing age [1-3]. The manifestations of SLE are associated with the presence of multiple autoantibodies $(\mathrm{Ab})$ that cause the formation and deposition of immune complexes (ICs), as well as other immune processes. Constitutional, muco-cutaneous and musculoskeletal signs represent the earliest and most common complaints reported by the majority of SLE patients. However, any organ, including the skin, hematologic, renal, neuropsychiatric (NP), cardiovascular and/or respiratory systems, can be affected. Not all manifestations perforce appear simultaneously, and a time interval of months or years may exist between the appearance of various symptoms [1]. As a consequence of this heterogeneous clinical presentation and pathogenesis, SLE is a disease that is still difficult to define. New classification criteria have been designed for research purposes but these cannot substitute for clinical judgment when making the diagnosis of SLE [4].

Despite recent improvements in the management of the disease, patients with lupus still have a high risk of morbidity and mortality. For example, in lupus nephritis (LN), nearly $10 \%$ of the patients progress towards end-stage kidney disease. Persistent disease activity, comorbidities and drug toxicity significantly contribute to the risk of progressive irreversible damage accrual and increased mortality $[5,6]$.

In the recent years, sustained remission has been proposed as the ultimate goal of the management of SLE, but it is rare to achieve [7]. Indeed, the Lupus Low Disease Activity State (LLDAS), which combines both low SLE activity and a low prednisone (PDN) doses $(\leq 7.5 \mathrm{mg}$ daily), is emerging as a more realistic target state [8]. The LLDAS is associated with a lower risk of new damage accrual and better health-related quality of life (HRQoL) [9, 10]

In summary, the therapeutic goals for patients with SLE have changed over the past 20 years. While in the first decades the focus was on patient survival, recent efforts have been directed towards the reduction of therapy-related side effects and organ damage with growing attention focused on HRQoL [11]. It is therefore crucial, in the context of a modern approach to the management of SLE, to combine global control of the disease with an adequate tolerability and safety of all available treatments.

This article is based on previously conducted studies and does not contain any studies with 
human participants or animals performed by any of the authors.

\section{CURRENT TREATMENT OPTIONS}

\section{Antimalarials}

Antimalarials are among the oldest drugs used to treat SLE, but they are still rightfully considered to be the cornerstone of SLE therapy. They are particularly effective in the management of skin manifestations and arthritis but should be included in the treatment regimen of every patient, unless there is a clear contraindication. The first use of antimalarial drugs in a patient with SLE probably occurred in 1894, when J.S. Payne described features of the lupus rash successfully treated with quinine. During the Second World War, quinacrine, used as malaria prophylaxis, improved various rheumatic complaints among soldiers. These observations led to studies on the use of antimalarial drugs in patients with rheumatic diseases, which demonstrated improvements in arthritis and cutaneous lupus among patients treated with quinacrine. Chloroquine was subsequently introduced in 1953, followed by hydroxychloroquine (HCQ) in 1955. HCQ showed the greatest efficacy and patient tolerability and became the most common antimalarial medication administered to patients with SLE [12]. In the 1990s, the role of HCQ in decreasing lupus flares, disease activity and glucocorticoid (GC) doses was further ascertained [13]. In 2007, Alarcon et al. showed that the use of HCQ improved survival in patients with SLE and was associated with a long-term protective effect on end-organ damage [14]; subsequent cohort studies have continued to support these findings. HCQ has also been found to exert pleiotropic favorable effects on endothelial dysfunction and the metabolic profile, thus reducing cardiovascular and thrombotic risk. In addition, HCQ has been shown to have a beneficial effect on pregnancy outcomes (including the prevention of congenital heart block), rates of infections and osteoporosis, and may ultimately play a role in preventing neoplasia [15]. Poor adherence or underdosing is the main issue associated with the use of HCQ, often due to concerns of retinal toxicity, which is, however, a rare complication that appears only after long-term treatment.

\section{Glucocorticosteroids}

The efficacy of GCs in the acute control of SLE is well established, and the use of high-dose or "pulsed" GCs to rapidly ablate the autoimmune response in organ-threatening manifestations is an important component of SLE treatment regimens. GCs were introduced in the 1950s for treating autoimmune diseases, with an overwhelming effect ("miracle drug"), and have contributed to an increase in the survival rates of patients with SLE [5]. However, soon after their introduction, it became clear that dosedependent side effects could occur in patients receiving GC treatment, and a series of studies subsequently demonstrated that their longterm use could have deleterious effects. In 2000, Zonana-Nacach and coworkers reported that GC use leads to permanent damage in multiple organ systems [16]. Gladman et al. found that up to $80 \%$ of the damage accrued was attributable to GC use [17], and a study by Thamer and colleagues showed that GC doses of 6-12 mg/day increase the risk of damage accrual to $50 \%$ [18]. Most studies have used 5-$7.5 \mathrm{mg}$ /day as a cut-off range, above which the risk for damage accrual considerably increases, but even low doses, over time, increase the risk for cataracts, osteoporosis, fractures and coronary artery disease [19].

\section{Conventional Immunosuppressive Agents}

The increasing concerns about the toxicity and severe side effects of GC have fostered the development of alternative therapeutic strategies. Conventional immunosuppressive (IS) drugs became a fundamental part of the therapeutic armamentarium for treating SLE. Along with their immunomodulatory properties, they also allow a more rapid and successful tapering of GC dose.

Early randomized controlled trials showed that combined therapy with GCs and 
cyclophosphamide (CYC), compared to GC monotherapy, led to a better renal outcome and to a higher remission rate in patients with $\mathrm{LN}$, but at the expense of a very high rate of infections and ovarian failure [20]. Houssiau et al. then conducted the pioneering Euro-Lupus Nephritis Trial (EuroLupus), which showed that low-dose CYC (cumulative dosage of $3 \mathrm{~g}$ ) was equally efficient for induction therapy of LN as the original high-dose CYC; thus low-dose CYC became the standard therapy for Caucasian patients with diffuse proliferative type III and type IV LN [21]. Other approaches to reduce treatment-related toxicities are based on the use of IS drugs, such as azathioprine (AZA), methotrexate (MTX), cyclosporine A (CsA) and mycophenolate mofetil (MMF). MMF has been shown not to be inferior to CYC for induction therapy of LN and even seems to be the preferable agent in African and Hispanic patients. Multi-target therapy has been recently proposed for induction therapy in LN [22]. In one study, the combination of tacrolimus with MMF and prednisolone (PDN) was superior to CYC and PDN when renal response was analyzed at 6 months, but not at 18 months [23]. In a further phase II trial, the addition of low-dose voclosporin, a new calcineurin inhibitor (CNI) with a more stable pharmacokinetic profile, to treatment with MMF resulted in a better renal response, but higher rates of adverse events, including death, were observed [24]. A phase III trial (AURORA) is ongoing.

MMF is considered to be the drug of choice for maintenance treatment, unless there is an ongoing pregnancy, in which case AZA is considered. CNIs and tacrolimus can also be used as useful adjunctive therapy in LN [22]. Among patients with non-renal disease, the choice of the IS drug is largely empirical: MTX when arthritis and cutaneous involvement are the predominant manifestations, and AZA or CsA in hematological disease or when pregnancy is contemplated. CYC is the treatment of choice for severe neuropsychiatric (NP) involvement in SLE and is also reserved for the treatment of any severe organ-threatening disease manifestations [25]. Sirolimus, an inhibitor of the mammalian target of rapamycin (mTOR), a serine-threonine kinase involved in T-cell proliferation, has recently emerged as a promising therapy in patients with clinically active SLE, particularly in those presenting musculoskeletal involvement $[26,27]$.

IS drugs are also burdened by their association to side effects, such as severe infections, malignancies, teratogenity and infertility, potentially leading to additional organ damage and mortality.

\section{Targeted Therapies: Biologic Agents}

In recent years, a better understanding of SLE etiopathogenesis has led to the introduction of a number of biologic agents that specifically target disease pathways underlying the development and progression of lupus. Some of these therapies, such as rituximab (RTX) and belimumab, are available in clinical practice, while others are being tested in ongoing clinical trials.

RTX is a chimeric monoclonal Ab which selectively targets B cell-specific surface molecule CD20. Two large, phase III, randomized placebo-controlled trials in non-renal lupus (EXPLORER) [28] and renal lupus (LUNAR) [29] failed to meet their primary endpoints; thus RTX still remains unlicensed. Despite the lack of trial evidence, the efficacy of RTX in the treatment of refractory LN and in severe non-renal SLE manifestations has been shown in many observational studies [30, 31]. The European League Against Rheumatism (EULAR) has recently stated that therapy with RTX should be considered in the treatment of organ-threatening, refractory lupus [32].

The only targeted biologic agent licensed for lupus to date is belimumab (Benlysta ${ }^{\circledR}$; GlaxoSmithKline), a fully humanized monoclonal antibody which inhibits B lymphocyte stimulator (BlyS), also known as B-cell activating factor (BAFF). Belimumab takes into account the pathophysiological significance of BAFF in autoimmune diseases. BAFF belongs to the tumor necrosis factor (TNF) family and was originally discovered in 1999 as a B-cell growth factor [33, 34]. Its central role in autoimmunity is well established. Elevated levels of BAFF correlate with autoimmune disease in humans, mice and dogs [35]. Transgenic over-expression 
of BAFF was found to lead to an accelerated lymphoproliferative disorder in mice, whereas BAFF-deficient mice were protected from SLE [33]. Two phase III trials, BLISS-52 and BLISS-76, studied the efficacy of intravenous belimumab on active SLE; both studies excluded patients with severe active $\mathrm{LN}$ and severe central nervous system (CNS) manifestations. A significant improvement of disease activity was achieved in the treatment arm compared with the placebo arm, and the trials thus met the primary endpoint. Belimumab also resulted in a reduction of flares and steroid use, as well as an improvement in HRQoL and levels of fatigue [36]. This led in 2011 to the approval of the use of belimumab to treat SLE $[37,38]$. The efficacy and safety of belimumab administered subcutaneously were confirmed in another trial [39]. Based on the results of randomized clinical trials (RCTs) and real-life experience, belimumab is particularly effective in patients with active disease, serological activity and high PDN intake, with earlier use achieving a better clinical response [40, 41]. The role of belimumab in $\mathrm{LN}$ is still being debated and will become clearer when the results of the ongoing BLISS-LN phase III RCT are published. In this context, a pooled post hoc analysis of the BLISS studies, with the aim to determine the effect of belimumab on patients with renal involvement, showed a greater improvement in renal disease in patients with serologic activity at baseline or receiving MMF, as has been confirmed in the real-life setting $[42,43]$. The finding of renal tubular epithelial cell-derived BAFF expression that mediated kidney damage and correlated with LN activity in the mouse and humans further supports the efficacy of belimumab in LN [44]. However, it must be noted that cases of new onset of LN in patients who received belimumab have been reported $[45,46]$, and preliminary retrospective observational data from a Swedish cohort showed increased risk and/or shorter time to de novo LN onset in non-renal patients undergoing therapy with belimumab [47]. Indeed, as the authors of these studies stated, the selection of patients suitable for belimumab therapy based on serological activity could be troublesome as these patients may be at high risk of developing $\mathrm{LN}$.
Regarding conventional IS, combination therapies designed to target two or more complementary pathways could be an effective strategy for the treatment of lupus, even with biologics. Two ongoing trials, BLISS-BELIEVE [48] and BEAT [49], are evaluating the efficacy of combined RTX and belimumab therapy. There is a strong rationale for this combined therapeutic approach. First, treatment with belimumab leads to the mobilization of memory B cells from tissues despite an overall decrease in peripheral B-cell levels, making tissue-resident B cells more susceptible to depletion by RTX. Second, blocking the effects of high BAFF levels in the serum might have favorable effects on B-cell reconstitution after depletion. The synergistic or additive effects of such a combination have been demonstrated in preclinical studies in lupus-prone mice and in case reports $[50,51]$.

\section{Adjuvant Treatments}

Intravenous immunoglobulins (IVIGs), which are purified from the plasma of healthy human donors, represent a valid therapeutic option for those patients with SLE with concomitant infections or for those who have contraindications or are refractory to conventional therapies. In some cases, for example in cases of neurological or hematological involvement, IVIGs can be given as first-line therapy [52]. Despite being used since the 1980s, IVIG therapy is still considered to be experimental without any clear indications. A systematic review of observational studies highlighted the association of IVIG administration with significant improvement in disease activity scores and complement levels [53].

Therapeutic plasma exchange (TPE) is a blood purification technique used for the removal of pathological substances, such as monoclonal para-proteins and auto- $\mathrm{Ab}$, as well as for the replacement of deficient plasma components. This therapeutic option is helpful when treatment with other agents fails or in presence of leucopenia and psychosis, but it has been profiled as an effective therapeutic option especially for patients with SLE with thrombotic 
thrombocytopenic purpura and catastrophic antiphospholipid syndrome [54].

In contrast with plasma exchange, immunoadsorption (IAS), a more specific form of extracorporeal treatment, leads to a specific and nearly complete clearance of circulating IgG and ICs, while neither removing other plasma proteins or necessitating substitution with fresh frozen plasma or albumin. In the past two decades IAS has emerged as a valuable option in the treatment of SLE, showing beneficial effects in patients with refractory disease or contraindications to standard immunosuppression, or during pregnancy [55]. Available studies have shown that short-term use of IAS reduces proteinuria and improves global disease activity, enabling GC dosages to be lowered [56]. In addition, prolonged IAS treatment has been shown to provide additional therapeutic benefits and to maintain an acceptable safety profile [55]. Additional evidence is needed to better define the clinical utility of IAS for specific SLE manifestations.

In the context of a holistic approach to lupus patients, there is an emerging body of evidence supporting the importance of correct lifestyle habits in the management of the disease, with particular attention to smoking cessation, physical activity and vitamin $\mathrm{D}$ supplementation [57]. According to the results of a recent meta-analysis, tobacco smoking, in addition to its well-known adverse effects, significantly reduces the effectiveness of HCQ and belimumab in cutaneous lesions and systemic manifestations. Cigarette smoking also appears to be a risk factor of SLE, negatively influencing the course of the disease [58]. Physical activity is emerging as pivotal in the reduction of cardiovascular risk and has also been shown to have positive effects on fatigue and mental health [59]. Vitamin D deficiency is common in patients with SLE, and a large body of evidence supports the negative impact of vitamin D deficiency on disease activity, fatigue and risk of thrombosis [57].

\section{EMERGING THERAPIES}

There are currently 125 registered studies on SLE that are recruiting participants; these include studies on targeting B cells, plasma cells, co-stimulation, cytokines and their receptors, chemokines and their receptors and complement factors or interferons. Clinical phase II or phase III RCTs on molecules targeting BAFF, such as tabalumab (IgG4 antibody to soluble and membrane BAFF), blisibimod (Fc fusion protein of 4 BAFF binding domains) or atacicept (APRIL [a proliferation-inducing ligand] and BAFF target) did not reach the respective endpoints [60-63]. Obinutuzumab (OBZ), a new-generation, glycoengineered type $\mathrm{II}$ anti-CD20 monoclonal Ab, induced superior B-cell cytotoxicity in patients with lupus in vitro, as demonstrated in whole blood assays [64], so further investigations on OBZ are ongoing.

B-cell receptor (BCR) signaling is believed to play a pivotal role in the development and maintenance of autoimmunity. The decrease of Lyn tyrosine kinase and spleen tyrosine kinase (Syk) in response to BCR stimulation in patients with active SLE further supports the hypothesis that $\mathrm{B}$ cells are under constant activation through BCR signaling [65]. Since it was thought that CD22 engagement would impose negative regulation of BCR signaling, epratuzumab, an anti-CD22 monoclonal antibody, was tested in SLE, but with negative results [66].

Other clinical approaches, such as with abatacept or CD40-CD40L, use T-/B-cell costimulatory pathways as the target. However, the phase II RCTs with abatacept in patients with SLE arthritis did not meet the primary endpoints $[67,68]$ and were stopped. CD40-CD40L blockade is currently being tested in patients with LN after a successful phase IIa RCT in patients with rheumatoid arthritis [69].

Long-lived plasma cells producing auto- $\mathrm{Ab}$ appear to be an interesting target of lupus research. As a consequence, a proteasome blocker, such as bortezomib, was used in a RCT, showing a good response [70]. However, there was a substantial rate of side effects [71]. Further 
clinical studies with other less toxic proteasome blockers are ongoing (e.g. ixazomib).

The Janus kinase/signal transducer and activator of transcription (JAK/STAT) pathway, which mediates the signal transduction of more than 50 cytokines and growth factors in many different cell types, has been found to have an emerging pathogenic relevance in SLE pathogenesis [72]. Baricitinib, a low-molecular-weight compound targeting JAK 1 and 2, met the primary endpoint at the dosage of $4 \mathrm{mg} /$ day in an international phase IIb clinical trial which involved patients with highly active SLE exhibiting skin and joint symptoms despite having received the standard treatment. The safety profile was consistent with that reported in previous studies, and serious infections were observed in a dose-dependent manner [73]. Baricitinib is currently under investigation in a phase III trial.

In recent years, one cytokine has been found to be of particular interest: type I interferon alpha (IFN $\alpha$ ) [74]. The accumulation of evidence showing that IFN-activated genes play a significant role in the pathophysiology of SLE has led to the testing of different therapeutic approaches. Although rontalizumab, a recombinant human monoclonal $\mathrm{Ab}$ against all 12 subtypes of IFN $\alpha$, did not meet the primary endpoint in a phase II clinical study, a subgroup analysis revealed positive effects that was dependent on the plasma IFN $\alpha$ levels [75]. The anti-IFN $\alpha$ receptor antibody anifrolumab has recently shown promising results $[76,77]$, leading to the good possibility that a second biologic for the therapy of active SLE will be approved in 2020. At the same time, this also means a step towards precision medicine, especially for SLE patients with a high IFNa activity who can benefit from this therapy.

Mitochondrial dysfunction recently emerged as a pivotal factor in the immune dysregulation and development of organ damage in SLE. Idebenone, a coenzyme Q10 synthetic quinone analog, has been found to ameliorate disease activity and the severity of organ damage, including glomerular inflammation and fibrosis in a murin model of SLE, suggesting a potential therapeutic role in humans [78]. Oxidative stress is increased in SLE, contributing to immune system dysregulation and comorbidities [79].
Reversal of glutathione depletion by application of its amino acid precursor, $\mathrm{N}$-acetylcysteine, safely improves disease activity in lupus patients by blocking mTOR in T lymphocytes, but further research is needed [80].

The loss of tolerance to self-antigens in SLE patients is associated with dysregulation of T-cell signaling, including the depletion of total levels of lymphocyte-specific protein kinase from sphingolipid-cholesterol-enriched membrane microdomains (lipid rafts). Atorvastatin has emerged as an effective therapy by targeting lipid raft-associated signaling abnormalities in autoreactive T cells [81].

Chimeric antigen receptor (CAR) T-cell therapy is an emergent approach in cancer immunotherapy and has shown efficacy in the treatment of B-cell malignancies through specific targeting of the B-cell surface antigen CD19 [82, 83]. CARs are fusion proteins consisting of a single-chain fragment from a monoclonal $\mathrm{Ab}$ specific for the target antigen of interest and T-cell receptor intracellular signaling domains. The treatment involves the isolation of a patient's autologous T cells, the genetic modification of these cells to express the antigen-specific CARs, cell expansion, and finally re-infusion of the modified cells into the patient [84]. Very recent data suggest that CAR T cells might also be a feasible strategy for the treatment of SLE. Using CD19-targeted CAR T cells, Kansal et al. showed sustained B-cell depletion in a murine lupus model [85]. Of note, CAR T cells targeting the BAFF receptor (BAFF-R) have proved to be effective in preclinical lymphoma and leukemia models [83]. Considering the encouraging results of current anti-BAFF therapies in SLE (reviewed above), BAFF-R-targeted CAR $T$ cells might be a promising novel therapeutic approach in patients suffering from SLE.

Interleukin-2 (IL-2) is involved in the expansion of immune tolerance by increasing regulatory $\mathrm{T}$ cells and suppressing effector $\mathrm{T}$ cells, including $\mathrm{T}$ helper 17 and $\mathrm{T}$ follicular helper cells. When used at high doses, IL-2 demonstrated efficacy in certain types of solid tumors, also contributing to the establishment of the concept of its use in cancer immunotherapy, while at low doses it was found to increase anti-infectious immune 
response [86]. A double-blind and placebo-controlled trial recently displayed the efficacy of low-dose IL-2 in the treatment of SLE, with a lower rate of infections, although not statistically significant, recorded in the IL-2 group compared to placebo [87].

The most relevant SLE therapies according to their different mechanisms of action are shown in Fig. 1.

\section{WHAT HAS ALREADY BEEN DONE AND WHAT REMAINS TO DO}

Advances in the treatment of SLE have been made in recent decades, leading to an increase in patient survival worldwide. Nevertheless, lupus currently is associated with a 2.4-fold increase in all-cause mortality. Patients with SLE have a higher risk of cardiovascular disease

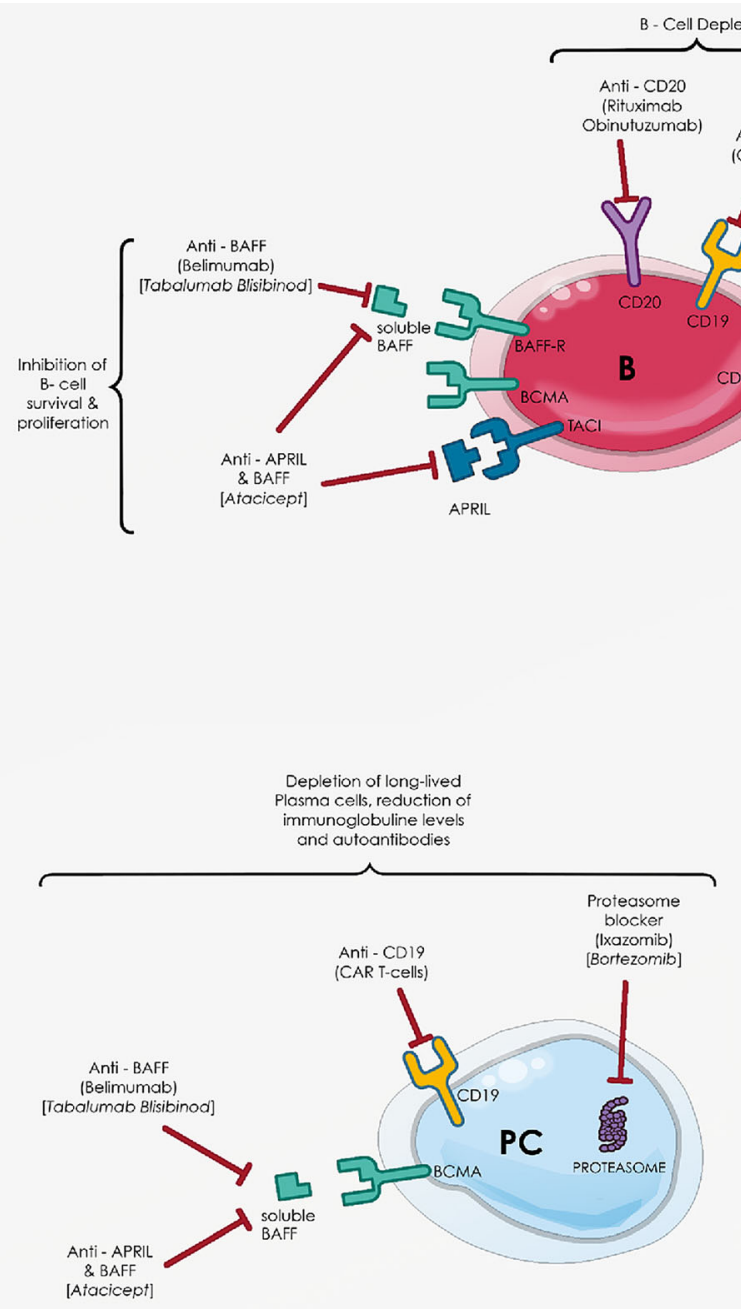

Fig. 1 Main mechanisms of systemic lupus erythematosus (lupus) pathogenesis with related targeted therapies. $B A F F$ B-cell-activating factor, $A P R I L$ a proliferation-inducing ligand, $B C M A$ B-cell maturation antigen, TACI

T-lymphocyte activation, $J A K$ Janus kinase, $T Y K 2$ tyrosine kinase 2, IFN- $\alpha$ interferon alpha, $I L-2$ interleukin-2, $H-2$ $\mathrm{T}$ helper 2, Treg regulatory $\mathrm{T}$ cells, CAR-T chimeric antigen receptor redirected 
(CVD) compared to the general population due to a complex interplay between traditional cardiovascular risk factors and SLE-specific conditions. CVD still represents the major cause of premature mortality in patients with SLE [5], but its effect remains underestimated among clinicians and treatment requires an interdisciplinary approach.

Patients with SLE also present a higher risk of infections, both as a consequence of their disease and of the therapies used in their clinical management. Live attenuated vaccines are contraindicated in patients receiving IS drugs, biologics and GCs at a dose $>10 \mathrm{mg}$ whereas inactivated vaccines can be used at any time. The risk of lupus flare deriving from vaccination has never been never confirmed, but vaccination rates remain low [88]. Further efforts are therefore needed to reinforce the immunization coverage of patients with SLE.

Lupus is known to cause a significant and sometimes severe neurological involvement, resulting in a number of diverse symptoms, referred to as NP-SLE. This heterogeneity in neurological and psychiatric symptoms occurring in SLE poses both diagnostic and therapeutic challenges with a substantial risk of either over- or under-treatment. It is well known that lupus patients with CNS involvement often present a more significant impairment of HRQoL when compared with those who have other serious manifestations of the disease (e.g. renal involvement) [1]. No decisive treatments for NP-SLE are as yet available, and there is only weak evidence on which to base recommendations [32].

Fatigue is the most common complaint of patients with SLE, reported by $80-100 \%$ of patients. It is the result of a multifactorial etiology and leads to a strong reduction of the HRQoL [89] and work productivity [90]. The relationship between several psychosocial factors, mainly pain, mood and sleep disorders, and comorbidities, such as fibromyalgia, anemia and vitamin D deficiency, is well established and can explain why fatigue is detectable even in patients in remission or with low disease activity. However, the relationship between fatigue and disease activity is still a matter of debate. We recently reported a significant correlation between the titer of the anti-NR2 $\mathrm{Ab}$, severity of fatigue, disease activity and anti-double stranded DNA Ab-independently from the presence of NP lupus manifestations. In addition, treatment with belimumab for at least 6 months affected both the severity of fatigue and the levels of anti-NR2 Ab [91]. This finding highlights NMDAR ( $N$-methyl-Daspartate) autoantibodies as a potential biomarker for fatigue in SLE and also provides a plausible explanation of belimumab efficacy in the treatment of fatigue, as observed in BLISS trials [36]. Physical activity and vitamin D supplementation [92] have also been shown to improve fatigue levels in patients with SLE. Despite these findings, relieving fatigue remains an unmet need for lupus patients and a challenge for their physicians, and new effective approaches are needed.

Over the last 30 years, advances in treatment have improved the life expectancy and HRQoL of SLE patients, but much more remains to be done. The heterogeneity of SLE, the different forms of progression and the concomitant medications have limited the approval of new therapies, despite major efforts of the scientific community and associated industries. A more precise characterization of disease phenotypes based on molecular and clinical features is expected to lead to the development of more effective and less toxic regimens in the management of SLE.

\section{ACKNOWLEDGEMENTS}

We wish to thank Vincenza Pellegrino for her contribution to the realization of Fig. 1.

Funding. We wish to thank the RARENET EU-Interreg for supporting the study. No Rapid Service Fee was received by the journal for the publication of this article.

Authorship. All named authors meet the International Committee of Medical Journal Editors (ICMJE) criteria for authorship for this article, take responsibility for the integrity of 
the work as a whole, and have given their approval for this version to be published.

Disclosures. Fabio Basta, Federica Fasola and Konstantinos Triantafyllias have nothing to disclose. Andreas Schwarting is a member of the Editorial Board of Rheumatology and Therapy.

Compliance with Ethical Guidelines. This article is based on previously conducted studies and does not contain any studies with human participants or animals performed by any of the authors.

Data Availability. Data sharing is not applicable to this article as no datasets were generated or analyzed during the current study.

Open Access. This article is licensed under a Creative Commons Attribution-NonCommercial 4.0 International License, which permits any non-commercial use, sharing, adaptation, distribution and reproduction in any medium or format, as long as you give appropriate credit to the original author(s) and the source, provide a link to the Creative Commons licence, and indicate if changes were made. The images or other third party material in this article are included in the article's Creative Commons licence, unless indicated otherwise in a credit line to the material. If material is not included in the article's Creative Commons licence and your intended use is not permitted by statutory regulation or exceeds the permitted use, you will need to obtain permission directly from the copyright holder. To view a copy of this licence, visit http://creativecommons.org/licenses/by$\mathrm{nc} / 4.0 /$.

\section{REFERENCES}

1. Tsokos GC. Systemic lupus erythematosus. N Engl J Med. 2011;365:2110-21.

2. Relle M, Foehr B, Schwarting A. Epigenetic aspects of systemic lupus erythematosus. Rheumatol Ther. 2015;2:33-46.
3. Bodis $G$, Toth $V$, Schwarting A. Role of human leukocyte antigens (HLA) in autoimmune diseases. Rheumatol Ther. 2018;5:5-20.

4. Aringer M, Costenbader K, Daikh D, et al. 2019 European League Against Rheumatism/American College of Rheumatology classification criteria for systemic lupus erythematosus. Ann Rheum Dis. 2019;78:1151-9.

5. Borchers AT, Keen CL, Shoenfeld Y, Gershwin ME. Surviving the butterfly and the wolf: mortality trends in systemic lupus erythematosus. Autoimmun Rev. 2004;3:423-53.

6. Frodlund M, Reid S, Wetterö J, Dahlström Ö, Sjöwall C, Leonard D. The majority of Swedish systemic lupus erythematosus patients are still affected by irreversible organ impairment: factors related to damage accrual in two regional cohorts. Lupus. 2019;28:1261-72.

7. Wilhelm TR, Magder LS, Petri M. Remission in systemic lupus erythematosus: durable remission is rare. Ann Rheum Dis. 2017;76:547-53.

8. Tani C, Vagelli R, Stagnaro C, Carli L, Mosca M. Remission and low disease activity in systemic lupus erythematosus: an achievable goal even with fewer steroids? Real-life data from a monocentric cohort. Lupus Sci Med. 2018;5:e000234.

9. Franklyn K, Lau CS, Navarra SV, et al. Definition and initial validation of a lupus low disease activity state (LLDAS). Ann Rheum Dis. 2016;75:1615-21.

10. Golder V, Kandane-Rathnayake R, Hoi AY, et al. Association of the lupus low disease activity state (LLDAS) with health-related quality of life in a multinational prospective study. Arthritis Res Ther. 2017;19:62.

11. Morand EF, Mosca M. Treat to target, remission and low disease activity in SLE. Best Pract Res Clin Rheumatol. 2017;31:342-50.

12. Lee SJ, Silverman E, Bargman JM. The role of antimalarial agents in the treatment of SLE and lupus nephritis. Nat Rev Nephrol. 2011;7:718-29.

13. Meinão IM, Sato EI, Andrade LE, Ferraz MB, Atra E. Controlled trial with chloroquine diphosphate in systemic lupus erythematosus. Lupus. 1996;5: 237-41.

14. Alarcón GS, McGwin G, Bertoli AM, et al. Effect of hydroxychloroquine on the survival of patients with systemic lupus erythematosus: data from LUMINA, a multiethnic US cohort (LUMINA L). Ann Rheum Dis. 2007;66:1168-72. 
15. Ponticelli C, Moroni G. Hydroxychloroquine in systemic lupus erythematosus (SLE). Expert Opin Drug Saf. 2017;16:411-9.

16. Zonana-Nacach A, Barr SG, Magder LS, Petri M. Damage in systemic lupus erythematosus and its association with corticosteroids. Arthritis Rheum. 2000;43:1801-8.

17. Gladman DD, Urowitz MB, Rahman P, Ibañez D, Tam L-S. Accrual of organ damage over time in patients with systemic lupus erythematosus. J Rheumatol. 2003;30:1955-9.

18. Thamer M, Hernán MA, Zhang Y, Cotter D, Petri M. Prednisone, lupus activity, and permanent organ damage. J Rheumatol. 2009;36:560-4.

19. Apostolopoulos D, Morand EF. It hasn't gone away: the problem of glucocorticoid use in lupus remains. Rheumatology (Oxford). 2017;56:i114-i12222.

20. Gourley MF, Austin HA 3rd, Scott D, et al. Methylprednisolone and cyclophosphamide, alone or in combination, in patients with lupus nephritis. A randomized, controlled trial. Ann Intern Med. 1996;125:549-57.

21. Houssiau FA, Vasconcelos C, D'Cruz D, et al. Immunosuppressive therapy in lupus nephritis: The Euro-Lupus Nephritis Trial, a randomized trial of low-dose versus high-dose intravenous cyclophosphamide. Arthritis Rheum. 2002;46:2121-31.

22. Parikh SV, Almaani S, Brodsky S, Rovin BH. Update on lupus nephritis: Core Curriculum 2020. Am J Kidney Dis. 2020. https://doi.org/10.1053/j.ajkd. 2019.10.017.

23. Liu Z, Zhang H, Liu Z, et al. Multitarget therapy for induction treatment of lupus nephritis: a randomized trial. Ann Intern Med. 2015;162:18-26.

24. Rovin BH, Solomons N, Pendergraft WF 3rd, et al. A randomized, controlled double-blind study comparing the efficacy and safety of dose-ranging voclosporin with placebo in achieving remission in patients with active lupus nephritis. Kidney Int. 2019;95:219-31.

25. Ugarte-Gil MF, Alarcón GS. Systemic lupus erythematosus: a therapeutic challenge for the XXI century. Clin Rheumatol. 2014;33:441-50.

26. Eriksson P, Wallin P, Sjöwall C. Clinical experience of sirolimus regarding efficacy and safety in systemic lupus erythematosus. Front Pharmacol. 2019;10:82.

27. Lai ZW, Kelly R, Winans T, et al. Sirolimus in patients with clinically active systemic lupus erythematosus resistant to, or intolerant of, conventional medications: a single-arm, open-label, phase 1/2 trial. Lancet. 2018;391:1186-96.

28. Merrill JT, Neuwelt CM, Wallace DJ, et al. Efficacy and safety of rituximab in moderately-to-severely active systemic lupus erythematosus: the randomized, double-blind, phase II/III systemic lupus erythematosus evaluation of rituximab trial. Arthritis Rheum. 2010;62:222-33.

29. Rovin BH, Furie R, Latinis K, et al. Efficacy and safety of rituximab in patients with active proliferative lupus nephritis: the Lupus Nephritis Assessment with Rituximab study. Arthritis Rheum. 2012;64:1215-26.

30. Witt M, Grunke M, Proft F, et al. Clinical outcomes and safety of rituximab treatment for patients with systemic lupus erythematosus (SLE) - results from a nationwide cohort in Germany (GRAID). Lupus. 2013;22:1142-9.

31. McCarthy EM, Sutton E, Nesbit S, et al. Short-term efficacy and safety of rituximab therapy in refractory systemic lupus erythematosus: results from the British Isles Lupus Assessment Group Biologics Register. Rheumatology (Oxford). 2018;57:470-9.

32. Fanouriakis A, Kostopoulou M, Alunno A, et al. 2019 update of the EULAR recommendations for the management of systemic lupus erythematosus. Ann Rheum Dis. 2019;78:736-45.

33. Mackay F, Woodcock SA, Lawton P, et al. Mice transgenic for BAFF develop lymphocytic disorders along with autoimmune manifestations. J Exp Med. 1999;190:1697-710.

34. Schneider P, MacKay F, Steiner V, et al. BAFF, a novel ligand of the tumor necrosis factor family, stimulates B cell growth. J Exp Med. 1999;189: 1747-56.

35. Vincent FB, Morand EF, Mackay F. BAFF and innate immunity: new therapeutic targets for systemic lupus erythematosus. Immunol Cell Biol. 2012;90: 293-303.

36. Strand V, Levy RA, Cervera R, et al. Improvements in health-related quality of life with belimumab, a B-lymphocyte stimulator-specific inhibitor, in patients with autoantibody-positive systemic lupus erythematosus from the randomised controlled BLISS trials. Ann Rheum Dis. 2014;73:838-44.

37. Navarra SV, Guzmán RM, Gallacher AE, et al. Efficacy and safety of belimumab in patients with active systemic lupus erythematosus: a randomised, placebo-controlled, phase 3 trial. Lancet. 2011;377: 721-31. 
38. Furie R, Petri M, Zamani O, et al. A phase III, randomized, placebo-controlled study of belimumab, a monoclonal antibody that inhibits B lymphocyte stimulator, in patients with systemic lupus erythematosus. Arthritis Rheum. 2011;63:3918-30.

39. Stohl W, Schwarting A, Okada M, et al. Efficacy and safety of subcutaneous belimumab in systemic lupus erythematosus: a fifty-two-week randomized, double-blind placebo-controlled study. Arthritis Rheumatol. 2017;69:1016-27.

40. Iaccarino L, Andreoli L, Bocci EB, et al. Clinical predictors of response and discontinuation of belimumab in patients with systemic lupus erythematosus in real life setting. Results of a large, multicentric, nationwide study. J Autoimmun. 2018;86:1-8.

41. Schwarting A, Schroeder JO, Alexander T, et al. First real-world insights into belimumab use and outcomes in routine clinical care of systemic lupus erythematosus in Germany: Results from the OBSErve Germany study. Rheumatol Ther. 2016;3: 271-90.

42. Dooley MA, Houssiau F, Aranow C, et al. Effect of belimumab treatment on renal outcomes: results from the phase 3 belimumab clinical trials in patients with SLE. Lupus. 2013;22:63-72.

43. Margiotta DPE, Basta F, Batani V, Afeltra A. Belimumab and low-doses of mycophenolate mofetil as induction therapy of class IV lupus nephritis: case series and literature review. BMC Nephrol. 2018;19: 54.

44. Schwarting A, Relle M, Meineck M, et al. Renal tubular epithelial cell-derived BAFF expression mediates kidney damage and correlates with activity of proliferative lupus nephritis in mouse and men. Lupus. 2018;27:243-56.

45. Sjöwall C, Cöster L. Belimumab may not prevent lupus nephritis in serologically active patients with ongoing non-renal disease activity. Scand J Rheumatol. 2014;43:428-30.

46. Staveri C, Karokis D, Liossis SC. New onset of lupus nephritis in two patients with SLE shortly after initiation of treatment with belimumab. Semin Arthritis Rheum. 2017;46:788-90.

47. Parodis I, Vital EM, Jönsen A, et al. De novo lupus nephritis during belimumab treatment. Poster presented at: 12th European Lupus Meeting. https:// doi.org/10.1136/lupus-2020-eurolupus.181.

48. Teng YKO, Bruce IN, Diamond B, et al. Phase III, multicentre, randomised, double-blind, placebocontrolled, 104-week study of subcutaneous belimumab administered in combination with rituximab in adults with systemic lupus erythematosus (SLE): BLISS-BELIEVE study protocol. BMJ Open. 2019;9:e025687.

49. Jones A, Muller P, Dore CJ, et al. Belimumab after B cell depletion therapy in patients with systemic lupus erythematosus (BEAT Lupus) protocol: a prospective multicentre, double-blind, randomised, placebo-controlled, 52-week phase II clinical trial. BMJ Open. 2019;9:e032569.

50. Bekar KW, Owen T, Dunn R, et al. Prolonged effects of short-term anti-CD20 B cell depletion therapy in murine systemic lupus erythematosus. Arthritis Rheum. 2010;62:2443-577.

51. Gualtierotti R, Borghi MO, Gerosa M, et al. Successful sequential therapy with rituximab and belimumab in patients with active systemic lupus erythematosus: a case series. Clin Exp Rheumatol. 2018;36:643-7.

52. Martínez T, Garcia-Robledo JE, Plata I, et al. Mechanisms of action and historical facts on the use of intravenous immunoglobulins in systemic lupus erythematosus. Autoimmun Rev. 2019;18:279-86.

53. Sakthiswary R, D'Cruz D. Intravenous immunoglobulin in the therapeutic armamentarium of systemic lupus erythematosus: a systematic review and meta-analysis. Medicine (Baltimore). 2014;93(16):e86.

54. Kronbichler A, Brezina B, Quintana LF, Jayne DR. Efficacy of plasma exchange and immunoadsorption in systemic lupus erythematosus and antiphospholipid syndrome: A systematic review. Autoimmun Rev. 2016;15:38-49.

55. Stummvoll GH, Aringer M, Smolen JS, et al. IgG immunoadsorption reduces systemic lupus erythematosus activity and proteinuria: a long term observational study. Ann Rheum Dis. 2005;64: 1015-21.

56. Stummvoll GH, Schmaldienst S, Smolen JS, Derfler K, Biesenbach P. Lupus nephritis: prolonged immunoadsorption (IAS) reduces proteinuria and stabilizes global disease activity. Nephrol Dial Transplant. 2012;27:618-26.

57. Guan SY, Cai HY, Wang P, et al. Association between circulating 25-hydroxyvitamin D and systemic lupus erythematosus: A systematic review and meta-analysis. Int J Rheum Dis. 2019;22: 1803-13.

58. Parisis D, Bernier C, Chasset F, Arnaud L. Impact of tobacco smoking upon disease risk, activity and therapeutic response in systemic lupus erythematosus: A systematic review and meta-analysis. Autoimmun Rev. 2019;18:102393. 
59. Margiotta DPE, Basta F, Dolcini G, et al. Physical activity and sedentary behavior in patients with systemic lupus erythematosus. PLoS ONE. 2018;13: e0193728.

60. Merrill JT, Shanahan WR, Scheinberg M, Kalunian KC, Wofsy D, Martin RS. Phase III trial results with blisibimod, a selective inhibitor of B-cell activating factor, in subjects with systemic lupus erythematosus (SLE): results from a randomised, doubleblind, placebo-controlled trial. Ann Rheum Dis. 2018;77:883-9.

61. Merrill JT, Wallace DJ, Wax S, et al. Efficacy and safety of atacicept in patients with systemic lupus erythematosus: results of a twenty-four-week, multicenter, randomized, double-blind, placebo-controlled, parallel-arm phase IIb study. Arthritis Rheumatol. 2018;70:266-76.

62. Merrill JT, van Vollenhoven RF, Buyon JP, et al. Efficacy and safety of subcutaneous tabalumab, a monoclonal antibody to B-cell activating factor, in patients with systemic lupus erythematosus: results from ILLUMINATE-2, a 52-week, phase III, multicentre, randomised, double-blind, placebo-controlled study. Ann Rheum Dis. 2016;75:332-40.

63. Isenberg DA, Petri M, Kalunian K, et al. Efficacy and safety of subcutaneous tabalumab in patients with systemic lupus erythematosus: results from ILLUMINATE-1, a 52-week, phase III, multicentre, randomised, double-blind, placebo-controlled study. Ann Rheum Dis. 2016;75:323-31.

64. Reddy V, Klein C, Isenberg DA, et al. Obinutuzumab induces superior B-cell cytotoxicity to rituximab in rheumatoid arthritis and systemic lupus erythematosus patient samples. Rheumatology (Oxford). 2017;56:1227-37.

65. Vásquez A, Baena A, González LA, et al. Altered recruitment of Lyn, Syk and ZAP-70 into lipid rafts of activated B cells in systemic lupus erythematosus. Cell Signal. 2019;58:9-19.

66. Clowse ME, Wallace DJ, Furie RA, et al. Efficacy and safety of epratuzumab in moderately to severely active systemic lupus erythematosus: results from two phase III randomized, double-blind placebocontrolled trials. Arthritis Rheumatol. 2017;69: 362-75.

67. Merrill JT, Burgos-Vargas R, Westhovens R, et al. The efficacy and safety of abatacept in patients with non-life-threatening manifestations of systemic lupus erythematosus: results of a twelve-month, multicenter, exploratory, phase IIb, randomized, double-blind, placebo-controlled trial. Arthritis Rheum. 2010;62:3077-87.
68. Furie R, Nicholls K, Cheng T-T, et al. Efficacy and safety of abatacept in lupus nephritis: a twelvemonth, randomized, double-blind study. Arthritis Rheumatol. 2014;66:379-89.

69. Visvanathan S, Daniluk S, Ptaszyński R, et al. Effects of BI 655064, an antagonistic anti-CD40 antibody, on clinical and biomarker variables in patients with active rheumatoid arthritis: a randomised, doubleblind, placebo-controlled, phase IIa study. Ann Rheum Dis. 2019;78:754-60.

70. Alexander T, Sarfert R, Klotsche J, et al. The proteasome inhibitior bortezomib depletes plasma cells and ameliorates clinical manifestations of refractory systemic lupus erythematosus. Ann Rheum Dis. 2015;74:1474-8.

71. Ishii $\mathrm{T}$, Tanaka $\mathrm{Y}$, Kawakami A, et al. Multicenter double-blind randomized controlled trial to evaluate the effectiveness and safety of bortezomib as a treatment for refractory systemic lupus erythematosus. Mod Rheumatol. 2018;28:986-92.

72. Alunno A, Padjen I, Fanouriakis, Boumpas DT. Pathogenic and therapeutic relevance of JAK/STAT signaling in systemic lupus erythematosus: integration of distinct inflammatory pathways and the prospect of their Inhibition with an oral agent. Cells. 2019;8(8):898. https://doi.org/10.3390/ cells8080898.

73. Wallace DJ, Furie RA, Tanaka Y, et al. Baricitinib for systemic lupus erythematosus: a double-blind, randomised, placebo-controlled, phase 2 trial. Lancet. 2018;392:222-31.

74. Weckerle CE, Franek BS, Kelly JA, et al. Network analysis of associations between serum interferon- $\alpha$ activity, autoantibodies, and clinical features in systemic lupus erythematosus. Arthritis Rheum. 2011;63:1044-53.

75. Kalunian KC, Merrill JT, Maciuca R, et al. A Phase II study of the efficacy and safety of rontalizumab (rhuMAb interferon- $\alpha$ ) in patients with systemic lupus erythematosus (ROSE). Ann Rheum Dis. 2016;75:196-202.

76. Furie R, Khamashta M, Merrill JT, et al. Anifrolumab, an anti-interferon- $\alpha$ receptor monoclonal antibody, in moderate-to-severe systemic lupus erythematosus. Arthritis Rheumatol. 2017;69: 376-86.

77. Morand EF, Furie R, Tanaka Y, et al. Trial of anifrolumab in active systemic lupus erythematosus. N Engl J Med. 2020;382:211-21.

78. Blanco LP, Pedersen HL, Wang X, et al. Improved mitochondrial metabolism and reduced inflammation following attenuation of murine lupus with 
coenzyme Q10 analog idebenone. Arthritis Rheumatol. 2020;72:454-64.

79. Perl A. Oxidative stress in the pathology and treatment of systemic lupus erythematosus. Nat Rev Rheumatol. 2013;9:674-86.

80. Lai ZW, Hanczko R, Bonilla E, et al. $N$-acetylcysteine reduces disease activity by blocking mammalian target of rapamycin in $\mathrm{T}$ cells from systemic lupus erythematosus patients: a randomized, doubleblind, placebo-controlled trial. Arthritis Rheum. 2012;64:2937-46.

81. Jury EC, Isenberg DA, Mauri C, Ehrenstein MR. Atorvastatin restores Lck expression and lipid raftassociated signaling in $\mathrm{T}$ cells from patients with systemic lupus erythematosus. J Immunol. 2006;177:7416-22.

82. Garfall AL, Maus MV, Hwang W-T, et al. Chimeric antigen receptor T cells against CD19 for multiple myeloma. N Engl J Med. 2015;373:1040-7.

83. Qin H, Dong Z, Wang X, et al. CAR T cells targeting BAFF-R can overcome CD19 antigen loss in B cell malignancies. Sci Transl Med. 2019;11:eaaw9414. https://doi.org/10.1126/scitranslmed.aaw9414.

84. Miliotou AN, Papadopoulou LC. CAR T-cell therapy: a new era in cancer immunotherapy. Curr Pharm Biotechnol. 2018;19:5-18.

85. Kansal R, Richardson N, Neeli I, et al. Sustained B cell depletion by CD19-targeted CAR T cells is a highly effective treatment for murine lupus. Sci Transl Med. 2019;1:eaav1648.

86. Klatzmann D, Abbas AK. The promise of low-dose interleukin-2 therapy for autoimmune and inflammatory diseases. Nat Rev Immunol. 2015;15: 283-94.

87. He J, Zhang R, Shao M, et al. Efficacy and safety of low-dose IL-2 in the treatment of systemic lupus erythematosus: a randomised, double-blind, placebo-controlled trial. Ann Rheum Dis. 2020;79: 141-9.

88. Grabar S, Groh M, Bahuaud M, et al. Pneumococcal vaccination in patients with systemic lupus erythematosus: A multicenter placebo-controlled randomized double-blind study. Vaccine. 2017;35: 4877-85.

89. Elefante E, Tani C, Stagnaro C, et al. Impact of fatigue on health-related quality of life and illness perception in a monocentric cohort of patients with systemic lupus erythematosus. RMD Open. 2020;6(1):e001133. https://doi.org/10.1136/ rmdopen-2019-001133.

90. Basta F, Margiotta DPE, Vadacca M, et al. Is fatigue a cause of work disability in systemic lupus erythematosus? Results from a systematic literature review. Eur Rev Med Pharmacol Sci. 2018;22: 4589-97.

91. Schwarting A, Möckel T, Lütgendorf F, et al. Fatigue in SLE: diagnostic and pathogenic impact of anti-Nmethyl-D-aspartate receptor (NMDAR) autoantibodies. Ann Rheum Dis. 2019;78:1226-344.

92. Zheng R, Gonzalez A, Yue J, et al. Efficacy and safety of vitamin D supplementation in patients with systemic lupus erythematosus: A meta-analysis of randomized controlled trials. Am J Med Sci. 2019;358:104-14. 